\title{
Evolution time and energy uncertainty
}

\author{
Timothy B Boykin ${ }^{1}$, Neerav Kharche ${ }^{2}$ \\ and Gerhard Klimeck ${ }^{2}$ \\ ${ }^{1}$ Department of Electrical and Compute Engineering, The University of Alabama in Huntsville, \\ Huntsville, AL 35899, USA \\ ${ }^{2}$ Network for Computational Nanotechnology, School of Electrical and Computer Engineering, \\ Purdue University, West Lafayette, IN 47907, USA
}

Received 13 April 2007

Published 23 May 2007

Online at stacks.iop.org/EJP/28/673

\begin{abstract}
Often one needs to calculate the evolution time of a state under a Hamiltonian with no explicit time dependence when only numerical methods are available. In cases such as this, the usual application of Fermi's golden rule and firstorder perturbation theory is inadequate as well as being computationally inconvenient. Instead, what one needs are conditions under which the evolution time may be obtained from the easily calculated energy uncertainty. This work derives some general conditions for obtaining the evolution time from the energy uncertainty.
\end{abstract}

\section{Introduction}

Time dependence is generally acknowledged to be one of the most subtle and difficult facets of quantum mechanics. The most detailed discussion of time-dependence in an undergraduate or beginner graduate course typically concentrates on Fermi's golden rule. Here one calculates the transition rate between initial and final states of the same, time-independent Hamiltonian to first order in response to a perturbation. A thorough discussion emphasizes that the time over which the perturbation acts must be of sufficient duration so that it can influence the system, yet must not exceed the intervals beyond which (1) the first-order treatment is valid; and (2) it becomes possible to resolve energy separations between the quasi-continuous set of final states. The transition rate problem is certainly important, yet there are other significant time-dependent problems which are often only glossed over.

One such issue is the more conceptually difficult energy-time uncertainty relation, which has been the topic of much recent research [1-3]. The problem most often studied involves the evolution of an initial state under a Hamiltonian which is not an explicit function of time. Hilgevoord [1] emphasizes that this relation is very different from the more familiar positionmomentum relation and Uffink [3] shows that it can be written in terms of the energy width of the distribution and the overlap of the initial and final wavefunctions. Gislason, Sabelli and Wood [2] discuss uncertainty relations and evolution times for several common distributions. 
These uncertainty relations connect the energy width of the distribution and the minimum time for the initial probability to have decayed to a given value.

The fact that the energy-time uncertainty relation establishes a lower limit on the evolution time leads to additional questions for the evolution of an initial state under a Hamiltonian with no explicit time dependence. What is the most meaningful definition of the evolution time for this case? Is it the time at which the probability to be found in the initial state drops below a certain value or is another definition more relevant? Can an expression for the evolution time (not merely a lower limit) be found and shown to hold, provided that the initial distribution satisfies some fairly general conditions? Can this evolution time be related to the energy uncertainty of the initial distribution? We emphasize that Fermi's golden rule cannot answer these questions since the basic physics of this problem is different. Here one is interested in the time required for the system to change sufficiently so it is no longer likely to find it in the initial state, as opposed to the transition rate between given initial and final states of the same Hamiltonian.

Peres [4] shows that the evolution time is approximately $\hbar / \Delta E$; however, the very general derivation employed makes checking the limits of validity difficult since the next higher term requires computing expectation values $\left\langle\hat{H}^{n}\right\rangle, n \geqslant 4$. One would like to have limits of validity expressed in terms of properties of the initial distribution, in much the same way that properties of the density of final states determine the applicability of Fermi's golden rule. In addition, the expression should be applicable to discrete distributions, which are often the only ones available from numerical calculations.

Here we provide a simple approximation for the evolution time in terms of the energy uncertainty and approximate limits of its validity. The treatment is formulated in terms of explicit states and is suitable for calculations where only numerical data are available. The physics of the problem leads us to a different definition of the evolution time from that employed previously [1-3] and we relate this time to the energy uncertainty.

\section{Evolution of the initial state and time-average probability}

The system studied has a Hamiltonian written as a sum of two time-independent parts with eigenstates and eigenvalues given by

$$
\begin{array}{ll}
\hat{H}=\hat{H}_{0}+\hat{H}_{1}, & {\left[\hat{H}_{0}, \hat{H}_{1}\right] \neq 0} \\
\hat{H}|n\rangle=\varepsilon_{n}|n\rangle, & \hat{H}_{0}|\nu\rangle=E_{v}|v\rangle .
\end{array}
$$

The two bases are related by the transformation

$$
|v\rangle=\sum_{n=1}^{N} c_{n}^{(v)}|n\rangle .
$$

The problem is to find the evolution time out of an initial state $|\psi(0)\rangle=|\nu\rangle$ under the full Hamiltonian, $\hat{H}$. That is, how long does it take for the system to evolve sufficiently so that there is little probability of finding it in the initial state? Note that this problem is fundamentally different from the typical transition rate calculation using Fermi's golden rule, where one is concerned with transitions from an initial state to a continuum of final states, all of which are eigenstates of the same Hamiltonian. Since the Hamiltonian has no explicit time dependence, the evolution operator is $\hat{U}(t, 0)=\exp [-\mathrm{i} t \hat{H} / \hbar]$ and

$|\psi(t)\rangle=\hat{U}(t, 0)|\psi(0)\rangle=\sum_{n=1}^{N} c_{n}^{(v)} \hat{U}(t, 0)|n\rangle=\sum_{n=1}^{N} c_{n}^{(v)} \exp \left[-\frac{\mathrm{i} \varepsilon_{n} t}{\hbar}\right]|n\rangle$. 
Equation (4) is used to find the energy uncertainty and the probability to be found in the initial state $|\psi(0)\rangle=|v\rangle$ at time $t$. These quantities, in turn, determine the evolution time.

The energy uncertainty, $\Delta E$, is obtained from a straightforward calculation using methods similar to those of [5] (details are given in the appendix):

$$
(\Delta E)^{2}=\frac{1}{2} \sum_{n=1}^{N} \sum_{m=1}^{N}\left(\varepsilon_{n}-\varepsilon_{m}\right)^{2}\left|c_{m}^{(v)}\right|^{2}\left|c_{n}^{(v)}\right|^{2}
$$

Note that $\Delta E$ is independent of time, as are $\langle\hat{H}\rangle$ and $\left\langle\hat{H}^{2}\right\rangle$.

The probability to be found in the original state, $\mathscr{P}_{v}(t)$, is calculated in a similar manner:

$$
\mathscr{P}_{\nu}(t)=|\langle\nu \mid \psi(t)\rangle|^{2}=\sum_{n=1}^{N} \sum_{m=1}^{N} \exp \left[-\frac{\mathrm{i}\left(\varepsilon_{n}-\varepsilon_{m}\right) t}{\hbar}\right]\left|c_{m}^{(v)}\right|^{2}\left|c_{n}^{(v)}\right|^{2} .
$$

Splitting and symmetrizing the double sum in equation (6) yields a convenient form:

$$
\begin{aligned}
\mathscr{P}_{\nu}(t)= & \frac{1}{2} \sum_{p=1}^{N} \sum_{q=1}^{N} \exp \left[-\frac{\mathrm{i}\left(\varepsilon_{p}-\varepsilon_{q}\right) t}{\hbar}\right]\left|c_{q}^{(v)}\right|^{2}\left|c_{p}^{(v)}\right|^{2} \\
& +\frac{1}{2} \sum_{p=1}^{N} \sum_{q=1}^{N} \exp \left[-\frac{\mathrm{i}\left(\varepsilon_{q}-\varepsilon_{p}\right) t}{\hbar}\right]\left|c_{q}^{(\nu)}\right|^{2}\left|c_{p}^{(v)}\right|^{2} \\
\mathscr{P}_{\nu}(t)= & \sum_{p=1}^{N} \sum_{q=1}^{N} \cos \left[\frac{\left(\varepsilon_{p}-\varepsilon_{q}\right) t}{\hbar}\right]\left|c_{p}^{(v)}\right|^{2}\left|c_{q}^{(v)}\right|^{2} .
\end{aligned}
$$

The presence of cosines in the expression for the probability to be found in the initial state, equation (8), implies that the probability is not necessarily a monotonically decreasing function of time. Indeed, if the energy differences are commensurate there is even a finite cycle time. More pertinently, however, the probability might decay significantly below unity only to rise rapidly back to near unity. This suggests that the minimum time after which the probability has decayed to a given small value, $\mathscr{P}_{e}$, is not the best definition of the evolution time. Instead, a definition which smoothes out the oscillations is more appropriate. We therefore calculate the average probability over a time $T$ :

$$
\overline{\mathscr{P}}_{\nu}(t)=\frac{1}{T} \int_{0}^{T} \mathscr{P}_{\nu}(t) \mathrm{d} t=\sum_{p=1}^{N} \sum_{q=1}^{N} \operatorname{sinc}\left[\frac{\left(\varepsilon_{p}-\varepsilon_{q}\right) T}{\hbar}\right]\left|c_{p}^{(v)}\right|^{2}\left|c_{q}^{(v)}\right|^{2},
$$

where $\operatorname{sinc}(x)=\sin (x) / x$. The evolution time, $T_{e}$, is therefore defined as the earliest time at which the average probability has decayed to an appropriate value, $\overline{\mathscr{P}}_{e}$.

To select an appropriate value for $\overline{\mathscr{P}}_{e}$, we note that for many physical systems, even those with oscillatory probabilities, the envelope of the probability generally decays. The most common example is that of exponential decay, where one lifetime of an exponentially decaying distribution is generally taken to be the evolution time. Accordingly, this observation suggests that a reasonable choice for $\overline{\mathscr{P}}_{e}$ would be the average of an exponentially decaying function over one lifetime:

$$
\overline{\mathscr{P}}_{e}=\frac{1}{\tau} \int_{0}^{\tau} \exp \left(-\frac{t}{\tau}\right) \mathrm{d} t \cong 0.6321 \approx \frac{2}{3} .
$$

Formally, then, the evolution time, $T_{e}$, is defined by the equation $\overline{\mathscr{P}}_{e}=\overline{\mathscr{P}}_{v}\left(T_{e}\right)$. Setting $\overline{\mathscr{P}}_{e}$ much higher is not physically reasonable. For the common case of exponential decay, solving

$$
\overline{\mathscr{P}}_{e}=\frac{1}{T} \int_{0}^{T} \exp \left(-\frac{t}{\tau}\right) \mathrm{d} t
$$


for $\overline{\mathscr{P}}_{e}=0.75$ yields $T \approx 0.61 \tau$, less than two-thirds of a lifetime. Later we will show that choosing too small a value for $\overline{\mathscr{P}}_{e}$ is likewise unreasonable.

\section{Approximate evolution time}

In addition to an appropriate value for the time-average probability, developing an expression for the approximate evolution time requires simplifying the sinc functions in equation (9). When the probability distribution, represented by $\left|c_{n}^{(v)}\right|^{2}$ is sharply peaked around some value, say $n=P$, a simplification is possible. Define an energy width, $W$, such that if $\left|\varepsilon_{n}-\varepsilon_{m}\right|>W$ the product $\left|c_{n}^{(v)}\right|^{2}\left|c_{m}^{(v)}\right|^{2}$ is small enough to be neglected. In this case the double sum in equation (9) is dominated by sinc functions with small arguments, each of which may be replaced by its Taylor expansion,

$$
\operatorname{sinc}(x) \approx 1-\frac{1}{6} x^{2}
$$

Note that the approximation, equation (11), is good to about $12 \%$ for $|x| \leqslant \sqrt{3}$, while for $|x|=2$ it is off by about $27 \%$. However, at a given time, $T$, larger $|x|$ occur where the product of coefficients $\left|c_{n}^{(v)}\right|^{2}\left|c_{m}^{(v)}\right|^{2}$ is smaller, making $|x| \leqslant 2$ a reasonable limit.

At this point Taylor expansion (12) could be used to directly obtain an approximate value for $T_{e}$. A more rigorous procedure involves finding upper and lower bounds on the evolution time, resulting in a better-controlled approximation. The idea of an upper bound on $T_{e}$ may at first seem odd. After all, typical discussions of uncertainty relations in quantum mechanics texts give only a lower bound: $\Delta E \Delta t \geqslant \hbar$. Upper bounds on time do, however, appear in thorough derivations of Fermi's golden rule.

Here, using the condition of a peaked probability distribution, which permits the requirement $|x| \leqslant 2$ in equation (12), an upper bound is easily found. Because $\operatorname{sinc}(2)-\left(1-2^{2} / 6\right)=\Delta_{2} \approx 0.12131538$, Taylor expansion (12) implies that the inequality

$$
1+\Delta_{2}-\frac{1}{6} x^{2} \geqslant \operatorname{sinc}(x), \quad 0 \leqslant x \leqslant 2
$$

holds. (This is easily demonstrated by graphing both functions.)

Substituting inequality (13) into equation (9), one finds

$$
\overline{\mathscr{P}}_{e} \leqslant\left(1+\Delta_{2}\right)\left[\sum_{p=1}^{N} \sum_{q=1}^{N}\left|c_{p}^{(v)}\right|^{2}\left|c_{q}^{(v)}\right|^{2}\right]-\frac{T_{e}^{2}}{6 \hbar^{2}} \sum_{p=1}^{N} \sum_{q=1}^{N}\left(\varepsilon_{p}-\varepsilon_{q}\right)^{2}\left|c_{p}^{(v)}\right|^{2}\left|c_{q}^{(v)}\right|^{2}
$$

The quantity in square brackets above is a unity, due to completeness, while the double sum in the second term is just $2(\Delta E)^{2}$, equation (5). Thus, using criterion (10), the evolution time satisfies the upper bound:

$$
T_{e} \leqslant \frac{\hbar}{\Delta E} \sqrt{3\left(1+\Delta_{2}-\overline{\mathscr{P}}_{\mathrm{e}}\right)} \approx 1.168 \frac{\hbar}{\Delta E} .
$$

Thus the evolution time has an approximate upper bound.

The more familiar lower bound on $T_{e}$ may be established using the Mandelstam-Tamm inequality (see Uffink [3] for a straightforward derivation):

$$
\mathscr{P}=|\langle\psi(0)|| \psi(t)\rangle|^{2} \geqslant \cos ^{2}\left(\frac{\Delta E t}{\hbar}\right), \quad 0 \leqslant t \leqslant \frac{\pi \hbar}{2 \Delta E} .
$$

Because both sides of inequality (16) are positive semi-definite over the interval of validity, we may integrate over an interval, $T$, to obtain the inequality satisfied by the time-average probability, $\overline{\mathscr{P}}_{e}$ :

$$
\overline{\mathscr{P}}_{e}=\frac{1}{T_{e}} \int_{0}^{T_{e}} \mathscr{P} \mathrm{d} t \geqslant \frac{1}{2}+\frac{1}{2} \operatorname{sinc}\left(\frac{2 \Delta E T_{e}}{\hbar}\right), \quad 0 \leqslant T_{e} \leqslant \frac{\pi \hbar}{2 \Delta E} .
$$


Observe that the upper limit on $T_{e}$ corresponds to the first zero of the sinc function. Inequality (17) immediately shows that demanding $\overline{\mathscr{P}}_{e}<1 / 2$ is meaningless for times satisfying $0 \leqslant T_{e} \leqslant \pi \hbar /(2 \Delta E)$, further justifying the choice $\overline{\mathscr{P}}_{e}=2 / 3$ in criterion (10). Because in this time range the sinc function is decreasing, a lower bound on $T_{e}$ follows from solving

$$
\operatorname{sinc}\left(\frac{2 \Delta E T_{e}^{(\min )}}{\hbar}\right)=2\left(\overline{\mathscr{P}}_{e}-\frac{1}{2}\right) \text {. }
$$

Doing so for $\overline{\mathscr{P}}_{e}=2 / 3$ yields $T_{e}^{(\min )} \approx 1.139431 \hbar / \Delta E$. Together with inequality (15) we then have limits on $T_{e}$ such that

$$
1.14 \frac{\hbar}{\Delta E} \leqslant T_{e} \leqslant 1.17 \frac{\hbar}{\Delta E}
$$

Note that these times are within the limits of the Mandelstam-Tamm inequality: $0 \leqslant$ $T_{e} \leqslant \pi \hbar /(2 \Delta E) \approx 1.57 \hbar / \Delta E$. We comment that selecting a slightly smaller value for the probability, say $\overline{\mathscr{P}}_{e}=7 / 12$, results in an incompatibility: $T_{e} \leqslant 1.27 \hbar / \Delta E$ and $T_{e} \geqslant 1.34 \hbar / \Delta E . \overline{\mathscr{P}}_{e} \approx 2 / 3$ thus appears to be close to the smallest value for which $T_{e}$ is compatible with the appropriate lower and upper limits.

\section{Short example}

A simple example illustrates the point. Take the energies to depend linearly on the index and a Gaussian form for the square coefficients:

$$
\varepsilon_{n}=n \varepsilon_{1}, \quad\left|c_{n}^{(v)}\right|^{2}=\mathscr{N} \exp \left[-\alpha^{2}(n-v)^{2}\right]
$$

where $\mathscr{N}$ is the normalization. In the example, we take $v=125, \alpha=0.15$. The 'exact' results are calculated with the first 700 terms of the series (no changes occur to five significant figures summing the first 1000 terms) and give $\Delta E_{\text {exact }}=4.714 \varepsilon_{1}$. The 'approximate' results ignore square coefficients $\left|c_{n}^{(v)}\right|^{2}<0.02 \max \left(\left|c_{n}^{(v)}\right|^{2}\right)$, with the maximum taken with respect to $n$. These give a window $W=14 \varepsilon_{1}$ (meaning that the sums extend over $111 \leqslant n \leqslant 139$ ) and an energy uncertainty $\Delta E_{\text {app }}=4.654 \varepsilon_{1}$. The 'exact' calculation gives $\bar{E}=125.00 \varepsilon_{1}$ while the 'approximate' calculation gives $\bar{E}=124.74 \varepsilon_{1}$. The uncertainties differ by about $1.3 \%$. To check the validity of equation (19), we set $T_{s}=1.15 \hbar / \Delta E$ (in the middle of the acceptable range) for both cases and calculated the resulting average probabilities, equation (9); the true answer is $2 / 3$ according to criterion (10). We find $\overline{\mathscr{P}}_{v}=0.6906$ (exact) and $\overline{\mathscr{P}}_{v}=0.6852$ (approximate). The approximate thus differs from the true value by about $2.8 \%$, which is reasonable in view of the approximation, equation (12).

\section{Conclusions}

We have thus found some general conditions under which the quantity $\hbar / \Delta E$ is a good estimate of the evolution time for an initial state under the action of a time-independent Hamiltonian. This value falls within the uncertainty relation derived by Uffink [3]. We have shown that defining the evolution time based on an average probability $\overline{\mathscr{P}}_{e}=2 / 3$ is physically reasonable and that, in this case, the evolution time satisfies $1.14 \hbar / \Delta E \leqslant T_{e} \leqslant 1.17 \hbar / \Delta E$. The evolution time we calculate here is not the conventionally employed minimum time at which the probability to be found in the initial state has decreased to a given value. Instead, our evolution time is the earliest time at which the time-averaged probability has fallen to a specific value, because using the time-average probability smoothes out possible oscillations. These results should be of special use in interpreting purely numerical calculations. 


\section{Acknowledgments}

The authors acknowledge the support of the Semiconductor Research Corporation. We thank K Balamukundhan and R Rahman for their comments.

\section{Appendix}

The expression for the standard deviation of energy can be obtained with methods similar to those of [5]. First, carry out the straightforward computations,

$$
\begin{aligned}
& \langle\hat{H}\rangle=\langle\psi(t)|\hat{H}| \psi(t)\rangle=\sum_{n=1}^{N} \varepsilon_{n}\left|c_{n}^{(v)}\right|^{2} \\
& \left\langle\hat{H}^{2}\right\rangle=\left\langle\psi(t)\left|\hat{H}^{2}\right| \psi(t)\right\rangle=\sum_{n=1}^{N} \varepsilon_{n}^{2}\left|c_{n}^{(v)}\right|^{2} \\
& (\Delta E)^{2}=\left\langle\hat{H}^{2}\right\rangle-\langle\hat{H}\rangle^{2}=\sum_{n=1}^{N} \varepsilon_{n}^{2}\left|c_{n}^{(v)}\right|^{2}-\sum_{n=1}^{N} \sum_{m=1}^{N} \varepsilon_{m} \varepsilon_{n}\left|c_{m}^{(v)}\right|^{2}\left|c_{n}^{(v)}\right|^{2}
\end{aligned}
$$

where equation (4) was used. Equation (A.3) is simplified by introducing the closure relation into the first sum to convert it to a double sum,

$$
\begin{gathered}
(\Delta E)^{2}=\sum_{n=1}^{N} \sum_{m=1}^{N} \varepsilon_{n}^{2}\left|c_{n}^{(v)}\right|^{2}\left|c_{m}^{(v)}\right|^{2}-\sum_{n=1}^{N} \sum_{m=1}^{N} \varepsilon_{m} \varepsilon_{n}\left|c_{m}^{(v)}\right|^{2}\left|c_{n}^{(v)}\right|^{2} \\
=\sum_{n=1}^{N} \varepsilon_{n} \sum_{m=1}^{N}\left(\varepsilon_{n}-\varepsilon_{m}\right)\left|c_{m}^{(\nu)}\right|^{2}\left|c_{n}^{(v)}\right|^{2}
\end{gathered}
$$

Equation (5) is obtained by symmetrizing equation (A.4), averaging double sums for which $\{n=p, m=q\}$ and $\{n=q, m=p\}$ :

$$
\begin{aligned}
& (\Delta E)^{2}=\frac{1}{2} \sum_{p=1}^{N} \varepsilon_{p} \sum_{q=1}^{N}\left(\varepsilon_{p}-\varepsilon_{q}\right)\left|c_{q}^{(v)}\right|^{2}\left|c_{p}^{(\nu)}\right|^{2}+\frac{1}{2} \sum_{q=1}^{N} \varepsilon_{q} \sum_{p=1}^{N}\left(\varepsilon_{q}-\varepsilon_{p}\right)\left|c_{p}^{(v)}\right|^{2}\left|c_{q}^{(v)}\right|^{2} \\
& (\Delta E)^{2}=\frac{1}{2} \sum_{p=1}^{N} \sum_{q=1}^{N}\left(\varepsilon_{p}-\varepsilon_{q}\right)^{2}\left|c_{q}^{(v)}\right|^{2}\left|c_{p}^{(\nu)}\right|^{2}
\end{aligned}
$$

\section{References}

[1] Hilgevoord J 1996 The uncertainty principle for energy and time Am. J. Phys. 64 1451-6

Hilgevoord J 1998 The uncertainty principle for energy and time: II Am. J. Phys. 66 396-402

Hilgevoord J 2002 Time in quantum mechanics Am. J. Phys. 70 301-6

[2] Gislason E A, Sabelli N H and Wood J W 1985 New form of the time-energy uncertainty relation Phys. Rev. A 31 2078-81

[3] Ufink J 1993 The rate of evolution of a quantum state Am. J. Phys. 61 935-6

[4] Peres A 1980 Zeno paradox in quantum theory Am. J. Phys. 48 931-2

[5] Scariano S M and Marlow A R 1991 An alternate way of computing uncertainties Am. J. Phys. 591149 